\title{
Relationship between the Predictability Limit and Initial Error in Chaotic Systems
}

\author{
Jianping Li and Ruiqiang Ding \\ State Key Laboratory of Numerical Modeling for Atmospheric Sciences and \\ Geophysical Fluid Dynamics, Institute of Atmospheric Physics, \\ Chinese Academy of Sciences, Beijing 100029
}

China

\section{Introduction}

Since the pioneer work of Lorenz on predictability problems [1-2], many studies have examined the relationships between predictability and initial error in chaotic systems [3-7]; however, these previous studies focused on multi-scale complex systems such as the atmosphere and oceans [4-6]. Because large uncertainties exist regarding the dynamic equations and observational data related to such complex systems, there also exists uncertainty in any conclusions drawn regarding the relationship between the predictability of such systems and initial error. In addition, multi-scale complex systems such as the atmosphere are thought to have an intrinsic upper limit of predictability due to interactions among different scales [2, 4-6]. The predictability time of multi-scale complex systems, regardless of the errors in initial conditions, cannot exceed their intrinsic limit of predictability.

For relatively simple chaotic systems with a single characteristic timescale driven by a small number of variables (e.g., the logistic map [7] and the Lorenz63 model [1]), their predictability limits continuously depend on the initial errors: the smaller the initial error, the greater the predictability limit. If the initial perturbation is of size $\delta_{0}$ and if the accepted error tolerance, $\Delta$, remains small, then the largest Lyapunov exponent $\Lambda_{1}$ gives a rough estimate of the predictability time: $T_{p} \sim \frac{1}{\Lambda_{1}} \ln \left(\frac{\Delta}{\delta_{0}}\right)$. However, reliance on the largest Lyapunov exponent commonly proves to be a considerable oversimplification [8]. This generally occurs because the largest Lyapunov exponent $\Lambda_{1}$, which we term the largest global Lyapunov exponent, is defined as the long-term average growth rate of a very small initial error. It is commonly the case that we are not primarily concerned with averages, and, even if we are, we may be interested in short-term behavior. Consequently, various local or finite-time Lyapunov exponents have been proposed, which measure the short-term growth rate of initial small perturbations [9-12]. However, the existing local or finite-time Lyapunov exponents, which are the same as the global Lyapunov exponent, are established based on the assumption that the initial perturbations are sufficiently small that their evolution can be approximately governed by the tangent linear model (TLM) of the nonlinear model, which essentially belongs to linear error dynamics. Clearly, as long as an uncertainty remains infinitesimal in the 
framework of linear error dynamics, it cannot pose a limit to predictability. To determine the limit of predictability, any proposed 'local Lyapunov exponent' must be defined with respect to the nonlinear behavior of nonlinear dynamical systems [13-14].

Recently, the nonlinear local Lyapunov exponent (NLLE) [15-17], which is a nonlinear generalization to the existing local Lyapunov exponents, was introduced to study the predictability of chaotic systems. NLLE measures the averaged growth rate of initial errors of nonlinear dynamical models without linearizing the governing equations. Using NLLE and its derivatives, the limit of dynamical predictability in large classes of chaotic systems can be efficiently and quantitatively determined. NLLE shows superior performance, compared with local or finite-time Lyapunov exponents based on linear error dynamics, in determining quantitatively the predictability limit of chaotic system. In the present study, we explore the relationship between the predictability limit and initial error in simple chaotic systems based on the NLLE approach, taking the logistic map and Lorenz63 model as examples.

\section{Nonlinear local Lyapunov exponent (NLLE)}

For an $n$-dimensional nonlinear dynamical system, its nonlinear perturbation equations are given by:

$$
\frac{d}{d t} \boldsymbol{\delta}(t)=\mathbf{J}(\mathbf{x}(t)) \boldsymbol{\delta}(t)+\mathbf{G}(\mathbf{x}(t), \boldsymbol{\delta}(t))
$$

where $\mathbf{x}(t)=\left(x_{1}(t), x_{2}(t), \cdots \cdots, x_{n}(t)\right)^{\mathrm{T}}$ is the reference solution, $\mathrm{T}$ is the transpose, $\mathbf{J}(\mathbf{x}(t)) \boldsymbol{\delta}(t)$ are the tangent linear terms, and $\mathbf{G}(\mathbf{x}(t), \boldsymbol{\delta}(t))$ are the high-order nonlinear terms of the perturbation $\delta(t)=\left(\delta_{1}(t), \delta_{2}(t), \cdots \cdots, \delta_{n}(t)\right)^{\mathrm{T}}$. Most previous studies have assumed that the initial perturbations are sufficiently small that their evolution could be approximately governed by linear equations [9-12]. However, linear error evolution is characterized by continuous exponential growth, which is not applicable to the description of a process that evolves from initially exponential growth to finally reaching saturation for sufficiently small errors (see Fig. 1). This linear approximation is also not applicable to situations in which the initial errors are not very small. Therefore, the nonlinear behaviors of error growth should be considered to determine the limit of predictability. Without linear approximation, the solutions of Eq. (1) can be obtained by numerical integration along the reference solution $\mathbf{x}(t)$ from $t=t_{0}$ to $t_{0}+\tau$ :

$$
\boldsymbol{\delta}\left(t_{0}+\tau\right)=\boldsymbol{\eta}\left(\mathbf{x}\left(t_{0}\right), \boldsymbol{\delta}\left(t_{0}\right), \tau\right) \boldsymbol{\delta}\left(t_{0}\right),
$$

where $\boldsymbol{\eta}\left(\mathbf{x}\left(t_{0}\right), \boldsymbol{\delta}\left(t_{0}\right), \tau\right)$ is the nonlinear propagator. NLLE is then defined as

$$
\lambda\left(\mathbf{x}\left(t_{0}\right), \boldsymbol{\delta}\left(t_{0}\right), \tau\right)=\frac{1}{\tau} \ln \frac{\left\|\boldsymbol{\delta}\left(t_{0}+\tau\right)\right\|}{\left\|\boldsymbol{\delta}\left(t_{0}\right)\right\|},
$$

where $\lambda\left(\mathbf{x}\left(t_{0}\right), \boldsymbol{\delta}\left(t_{0}\right), \tau\right)$ depends in general on the initial state $\mathbf{x}\left(t_{0}\right)$ in phase space, the initial error $\delta\left(t_{0}\right)$, and time $\tau$. This differs from the existing local or finite-time Lyapunov exponents, which are defined based on linear error dynamics [9-12]. In the case of double limits of $\left\|\boldsymbol{\delta}\left(t_{0}\right)\right\| \rightarrow 0$ and $\tau \rightarrow \infty$, NLLE converges to the largest global Lyapunov exponent $\Lambda_{1}$. The ensemble mean NLLE over the global attractor of the dynamical system is given by 


$$
\begin{aligned}
& \bar{\lambda}\left(\boldsymbol{\delta}\left(t_{0}\right), \tau\right)=\int_{\Omega} \lambda\left(\mathbf{x}\left(t_{0}\right), \boldsymbol{\delta}\left(t_{0}\right), \tau\right) d \mathbf{x} \\
& =\left\langle\lambda\left(\mathbf{x}\left(t_{0}\right), \boldsymbol{\delta}\left(t_{0}\right), \tau\right)\right\rangle_{N},(N \rightarrow \infty)
\end{aligned}
$$

where $\Omega$ represents the domain of the global attractor of the system, and \langle\rangle$_{N}$ denotes the ensemble average of samples of sufficiently large size $N(N \rightarrow \infty)$. The mean relative growth of initial error (RGIE) can be obtained by

$$
\bar{E}\left(\boldsymbol{\delta}\left(t_{0}\right), \tau\right)=\exp \left(\bar{\lambda}\left(\boldsymbol{\delta}\left(t_{0}\right), \tau\right) \tau\right) .
$$

Using the theorem from Ding and Li [16], then we obtain

$$
\bar{E}\left(\boldsymbol{\delta}\left(t_{0}\right), \tau\right) \stackrel{P}{\longrightarrow} \mathcal{C}(N \rightarrow \infty)
$$

where $\stackrel{P}{\longrightarrow}$ denotes the convergence in probability and $c$ is a constant that depends on the converged probability distribution of error growth $P$. This is termed the saturation property of RGIE for chaotic systems. The constant $c$ can be considered as the theoretical saturation level of $\bar{E}\left(\boldsymbol{\delta}\left(t_{0}\right), \tau\right)$. Once the error growth reaches the saturation level, almost all information on initial states is lost and the prediction becomes meaningless. Using the theoretical saturation level, the limit of dynamical predictability can be determined quantitatively [15-16]. In addition, for $\bar{\lambda}\left(\boldsymbol{\delta}\left(t_{0}\right), \tau\right)=\frac{1}{t} \ln \left[\bar{E}\left(\boldsymbol{\delta}\left(t_{0}\right), \tau\right)\right]$, based on the above analysis, we have

$$
\bar{\lambda}\left(\boldsymbol{\delta}\left(t_{0}\right), \tau\right) \stackrel{P}{\longrightarrow} \frac{1}{\tau} \times \ln c \text { as } \tau \rightarrow \infty ;
$$

therefore, $\bar{\lambda}\left(\boldsymbol{\delta}\left(t_{0}\right), \tau\right)$ asymptotically decreases like $\mathrm{O}(1 / \tau)$ as $\tau \rightarrow \infty$. The magnitude of the initial error $\delta_{0}$ is defined as the norm of the vector error $\boldsymbol{\delta}\left(t_{0}\right)$ in phase space at the initial time $t_{0}$; i.e., $\delta_{0}=\left\|\boldsymbol{\delta}\left(t_{0}\right)\right\|$. The results show that the limit of dynamical predictability depends mainly on the magnitude of the initial error $\delta\left(t_{0}\right)$ and rather than on its direction, because the error direction in the phase space becomes rapidly aligned toward the most unstable direction (Fig. 2).

\section{Experimental predictability results}

The first example is the logistic map [7],

$$
y_{n+1}=a y_{n}\left(1-y_{n}\right), 0 \leq a \leq 4,
$$

Here, we choose the parameter value of $a=4.0$, for which the logistic map is chaotic on the set $(0,1)$ [18-19]. Figure 3 shows the dependence of the mean NLLE and the mean RGIE on the magnitude of the initial error. The mean NLLE is essentially constant in a plateau region that widens as decreasing initial error $\delta_{0}$ (Fig. 3a). For a sufficiently long time, however, all the curves are asymptotic to zero. This finding reveals that for a very small initial error, 
error growth is initially exponential, with a growth rate consistent with the largest global Lyapunov exponent, indicating that linear error dynamics are applicable during this phase. Subsequently, the error growth enters a nonlinear phase with a steadily decreasing growth rate, finally reaching a saturation value.

Figure $3 \mathrm{~b}$ shows that the time at which the error growth reaches saturation also lengthens as $\delta_{0}$ is reduced. Regardless of the magnitude of the initial error $\delta_{0}$, all the errors ultimately reach saturation. To estimate the predictability time of a chaotic system, the predictability limit is defined as the time at which the error reaches $99 \%$ of its saturation level. The limit of dynamic predictability is found to decrease approximately linearly as increasing logarithm of initial error (Fig. 4). For a specific initial error, the limit of dynamic predictability is longer than the time for which the tangent linear approximation holds, which is defined as the time over which the mean NLLE remains constant. The difference between the predictability limit and the time over which the tangent linear approximation holds, remains largely constant as increasing logarithm of initial error, suggesting that the time over which the nonlinear phase of error growth lasts may be constant for initial errors of various magnitudes.

The second example is the Lorenz63 model [1],

$$
\left\{\begin{array}{l}
\dot{X}=-\sigma X+\sigma Y \\
\dot{Y}=r X-Y-X Z \\
\dot{Z}=X Y-b Z
\end{array}\right.
$$

where $\sigma=10, r=28$, and $b=8 / 3$, for which the well-known "butterfly" attractor exists. Figure 5 shows the mean NLLE and mean RGIE with initial errors of various magnitudes as a function of time $\tau$. For all initial errors, the mean NLLE is initially unstable, then remains constant and finally decreases rapidly, approaching zero as increasing $\tau$ (Fig. 5a). For a very small initial error, it does not take long for error growth to become exponential, with a growth rate consistent with the largest global Lyapunov exponent, indicating that linear error dynamics are applicable during this phase. Subsequently, error growth enters a nonlinear phase with a steadily decreasing growth rate, finally reaching a saturation value (Fig. 5b). For initial errors of various magnitudes, the predictability limit of the Lorenz63 model is defined as the time at which the error reaches $99 \%$ of its saturation level, similar to the case for the logistic map.

Figure 6 shows the predictability limit and the time over which the tangent linear approximation holds as a function of the magnitude initial error. The predictability limit of the Lorenz63 model decreases approximately linearly as increasing logarithm of initial error, similar to the logistic map. For the Lorenz63 model, the difference between the predictability limit and the time over which the tangent linear approximation holds, remains largely constant as increasing logarithm of initial error.

\section{Theoretical predictability analysis}

As shown above, there exists a linear relationship between the predictability limit and the logarithm of initial error, for both the logistic map and Lorenz63 model. To understand the reason for this linear relationship, it is necessary to further investigate the relationship between the predictability limit and the logarithm of initial error using the theoretical 
analysis, to determine if a general law exists between the predictability limit and the logarithm of initial error for chaotic systems.

For relatively simple chaotic systems such as the logistic map and Lorenz63 model, the predictability limit $T_{p}$ is assumed to consist of the following two parts:

$$
T_{p}=T_{L}+T_{N}
$$

where $T_{L}$ is the time over which the tangent linear approximation holds, and $T_{N}$ is the time over which the nonlinear phase of error growth occurs. When the mean error reaches a critical value $\delta_{c}$, which is thought to be almost constant for a chaotic system under the condition of the given parameters, the tangent linear approximation is no longer valid and the error growth enters the nonlinear phase. Under the condition of the given parameters, the saturation value of error $E^{*}$ is constant, which is not dependent on the initial error. Consequently, the time $T_{N}$ taken for the error growth from $\delta_{c}$ to $E^{*}$ can be considered as almost constant, not relying on the initial error. This assumption is confirmed by the experimental results shown in Figs. 3 and 5, which indicate that the interval between the predictability limit and the time over which the tangent linear approximation holds, remains almost constant as increasing logarithm of initial error. Then, $T_{N}$ can be written as a constant:

$$
T_{N}=C_{1}
$$

For $T_{L}$, the time over which the tangent linear approximation holds, we get

$$
\delta_{c}=\delta_{0} \exp \left(\Lambda_{1} T_{L}\right) \text {, }
$$

where $\delta_{0}$ is the initial error and $\Lambda_{1}$ is the largest global Lyapunov exponent. From Eq. (12), we have

$$
T_{L}=\frac{1}{\Lambda_{1}} \ln \left(\frac{\delta_{c}}{\delta_{0}}\right) .
$$

From Eqs. (10), (11), and (13), we obtain

$$
T_{p}=C_{1}+\frac{1}{\Lambda_{1}}\left[\ln \delta_{c}-\ln \delta_{0}\right]
$$

Under the condition of the given parameters, $\Lambda_{1}$ of the chaotic system is fixed, as is $\frac{1}{\Lambda_{1}} \ln \delta_{c}$. Then, we have $\frac{1}{\Lambda_{1}} \ln \delta_{c}=C_{2}$ (where $C_{2}$ is a constant). Therefore, $T_{p}$ can be written as

$$
T_{p}=C-\frac{1}{\Lambda_{1}} \ln \delta_{0},
$$

where $C=C_{1}+C_{2}$. Eq. (15) can be changed to 


$$
T_{p}=C-\frac{1}{\Lambda_{1}} \frac{\log _{10} \delta_{0}}{\log _{10} e} .
$$

If the largest global Lyapunov exponent $\Lambda_{1}$ and the constant $C$ are known in advance, the predictability limit can be obtained for initial errors of any magnitude, according to Eq. (16). The constant $C$ can be calculated from Eq. (16) if the predictability limit corresponding to a fixed initial error has been obtained in advance through the NLLE approach.

\section{Experimental verification of theoretical results}

Using the method proposed by Wolf et al. [20], the largest global Lyapunov exponent $\Lambda_{1}$ of the logistic map is 0.693 when $a=4.0$. From Eq. (16), we have the formula that describes the relationship between the predictability limit and the initial error of the logistic map:

$$
T_{p}=C-3.32 \log _{10} \delta_{0}
$$

For $\delta_{0}=10^{-6}$, the predictability limit of the logistic map is $T_{p}=18$, as obtained using the NLLE approach. Then, we have $C=-1.92$ in Eq. (17). Therefore, the predictability limit for various initial errors can be obtained from Eq. (17). The predictability limits obtained in this way are in good agreement with those obtained using the NLLE approach (Fig. 7). This finding indicates that the assumptions presented in Section 3 are indeed reasonable. Therefore, it is appropriate to determine the predictability limit of the logistic map by extrapolating Eq. (17) to various initial errors.

The largest global Lyapunov exponent $\Lambda_{1}$ of the Lorenz63 model is obtained to be 0.906 when $\sigma=10, r=28, b=8 / 3$. From Eq. (16), we have the formula that describes the relationship between the predictability limit and the initial error of the Lorenz63 model:

$$
T_{p}=C-2.54 \log _{10} \delta_{0}
$$

For $\delta_{0}=10^{-6}$, the predictability limit of the Lorenz63 model is $T_{p}=22.19$, as obtained using the NLLE approach. Then, we have $C=6.95$ in Eq. (17). Therefore, the predictability limits for various initial errors can be obtained by extrapolating the Eq. (17) to various initial errors. The resulting limits are in good agreement with those obtained using the NLLE approach (Fig. 8). The linear relationship between the predictability limit and the logarithm of initial error is further verified by the Lorenz63 model, and the relationship may be applicable to other simple chaotic systems.

\section{Summary}

Previous studies that examine the relationship between predictability and initial error in chaotic systems with a single characteristic timescale were based mainly on linear error dynamics, which were established based on the assumption that the initial perturbations are sufficiently small that their evolution could be approximately governed by the TLM of the nonlinear model. However, linear error dynamics involves large limitations, which is not applicable to determine the predictability limit of chaotic systems. 
Taking the logistic map and Lorenz63 model as examples, we investigated the relationship between the predictability limit and initial error in chaotic systems, using the NLLE approach, which involves nonlinear error growth dynamics. There exists a linear relationship between the predictability limit and the logarithm of initial error. A theoretical analysis performed under the nonlinear error growth dynamics revealed that the growth of mean error enters a nonlinear phase after it reaches a certain critical magnitude, finally reaching saturation. For a given chaotic system, if the control parameters of the system are given, then the saturation value of error growth is fixed. The time taken for error growth from the nonlinear phase to saturation is also almost constant for various initial errors. The predictability limit is only dependent on the phase of linear error growth. Consequently, there exists a linear relationship between the predictability limit and the logarithm of initial error. The linear coefficient is related to the largest global Lyapunov exponent: the greater the latter, the more rapidly the predictability limit decreases as increasing logarithm of initial error. If the largest global Lyapunov exponent and the predictability limit corresponding to a fixed initial error are known in advance, the predictability limit can be extrapolated to various initial errors based on the linear relationship between the predictability limit and the logarithm of initial error.

It should be noted that the linear relationship between the predictability limit and the logarithm of initial error holds only in the case of relatively small initial errors. If the initial errors are large, the growth of the mean error would directly enter into the nonlinear phase, meaning that the linear relationship would fail to describe the relationship between the predictability limit and the logarithm of initial error. A more complex relationship may exist between the predictability limit and initial errors, which is an important subject left for future research.

\section{Acknowledgment}

This research was funded by an NSFC Project (40805022) and the 973 program (2010CB950400).

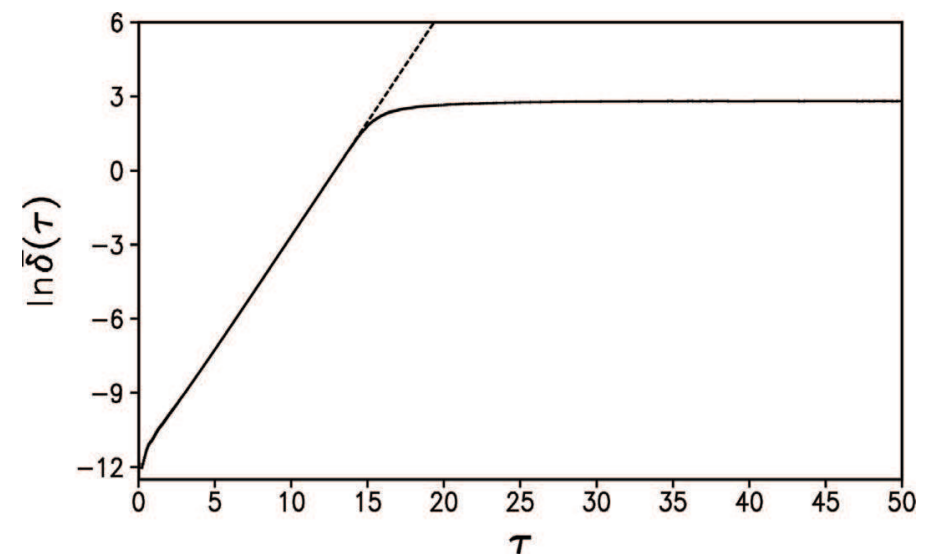

Fig. 1. Linear (dashed line) and nonlinear (solid line) average growth of errors in the Lorenz system as a function of time. The initial magnitude of errors is $10^{-5}$. 

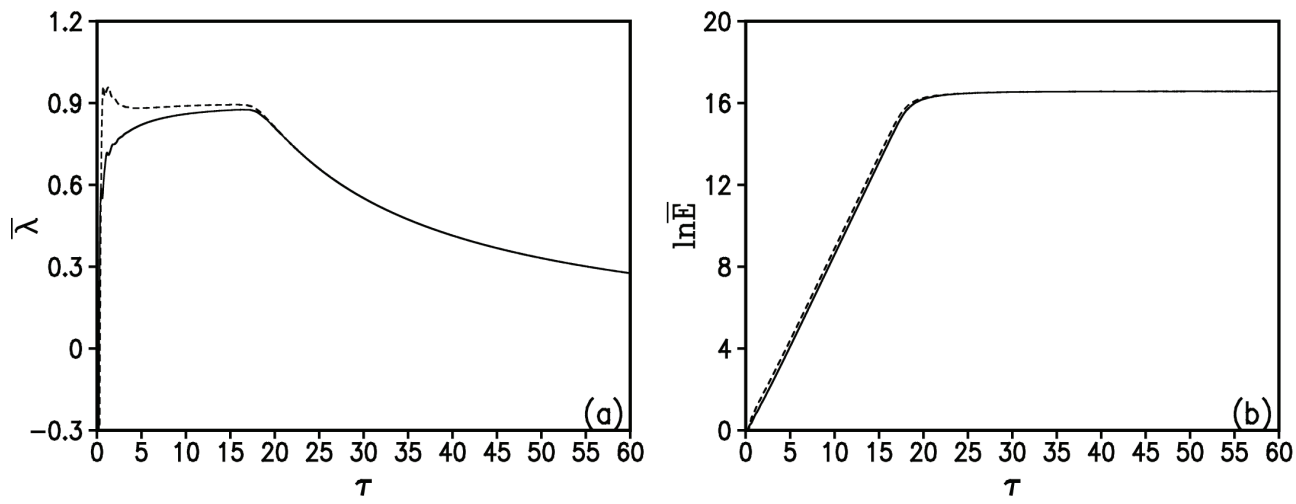

Fig. 2. Mean NLLE $\bar{\lambda}\left(\boldsymbol{\delta}\left(t_{0}\right), \tau\right)$ (a) and the logarithm of the corresponding mean RGIE $\bar{E}\left(\boldsymbol{\delta}\left(t_{0}\right), \tau\right)(\mathrm{b})$ in the Lorenz63 model as a function of time $\tau$. In (a) and (b), the dashed and solid lines correspond to the initial errors $\boldsymbol{\delta}\left(t_{0}\right)=\left(10^{-6}, 0,0\right)$ and $\boldsymbol{\delta}\left(t_{0}\right)=\left(0,0,10^{-6}\right)$, respectively.
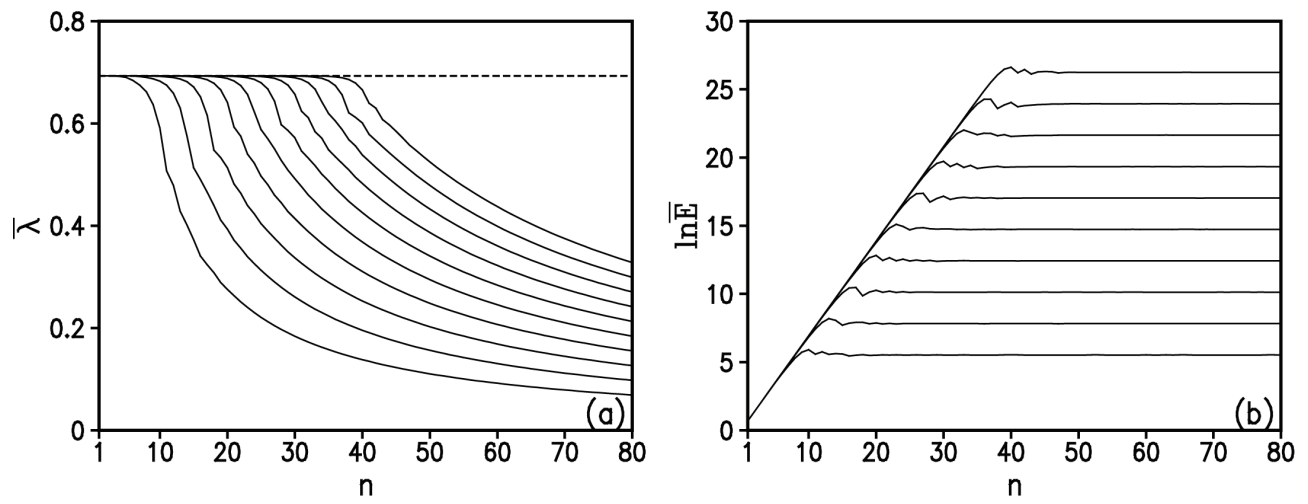

Fig. 3. Mean NLLE $\bar{\lambda}\left(\boldsymbol{\delta}\left(t_{0}\right), n\right)$ (a) and the logarithm of the corresponding mean RGIE $\bar{E}\left(\boldsymbol{\delta}\left(t_{0}\right), n\right)(\mathrm{b})$ in the logistic map as a function of time step $n$ and $\delta_{0}$ of various magnitudes. From above to below, the curves correspond to $\delta_{0}=10^{-12}, 10^{-11}, 10^{-10}, 10^{-9}, 10^{-8}$, $10^{-7}, 10^{-6}, 10^{-5}, 10^{-4}$, and $10^{-3}$, respectively. In (a), the dashed line indicates the largest global Lyapunov exponent. 


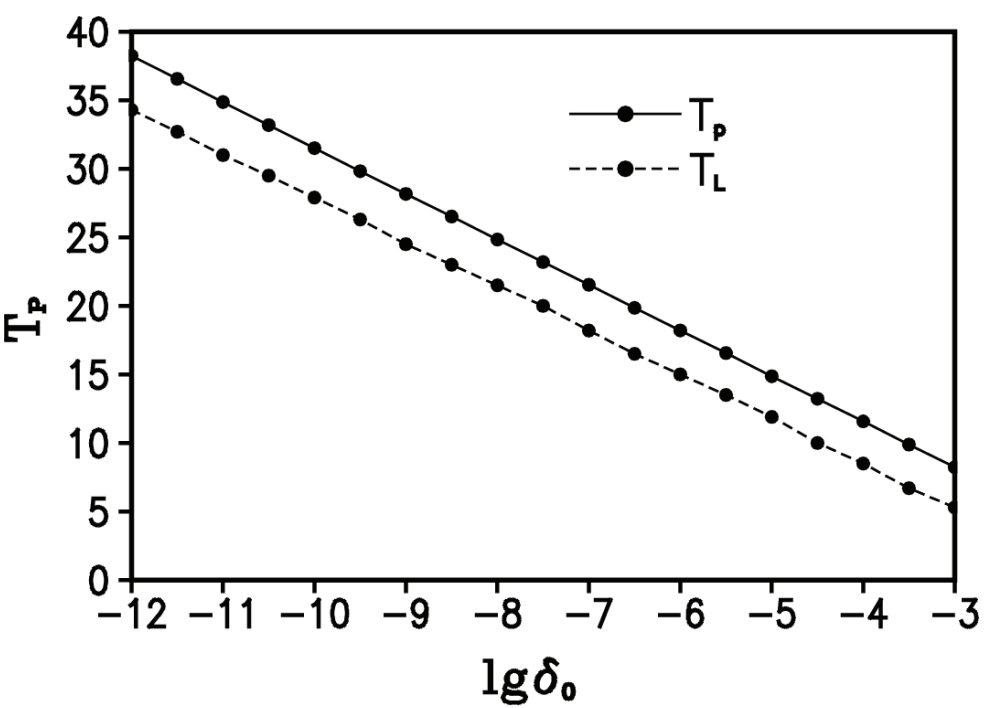

Fig. 4. Predictability limit $T_{P}$ and the time $T_{L}$ over which the tangent linear approximation holds in the logistic map as a function of $\delta_{0}$ of various magnitudes.
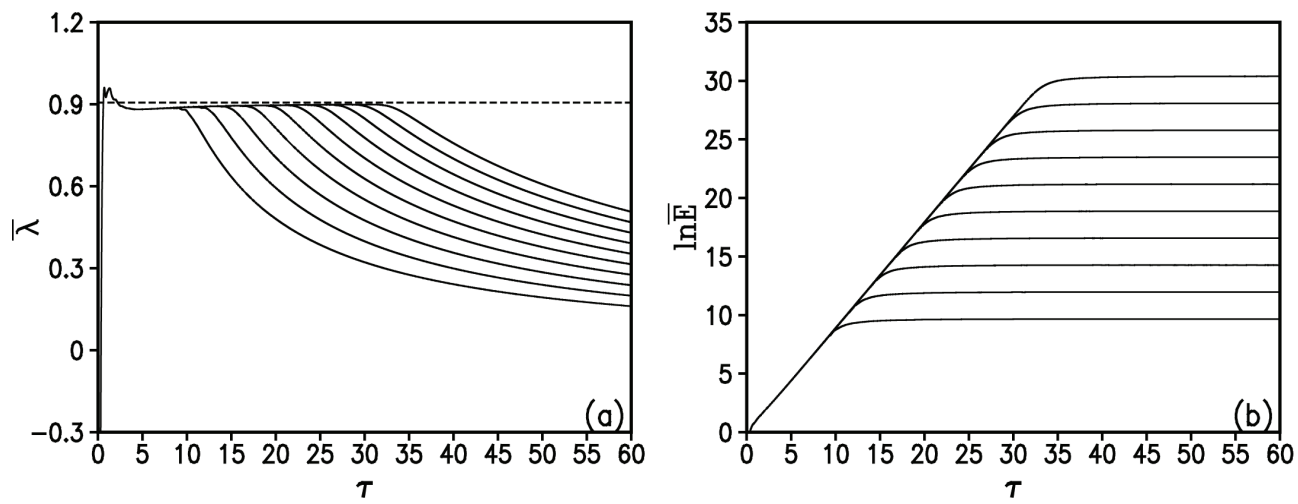

Fig. 5. Same as Fig. 3, but for the Lorenz63 model. 


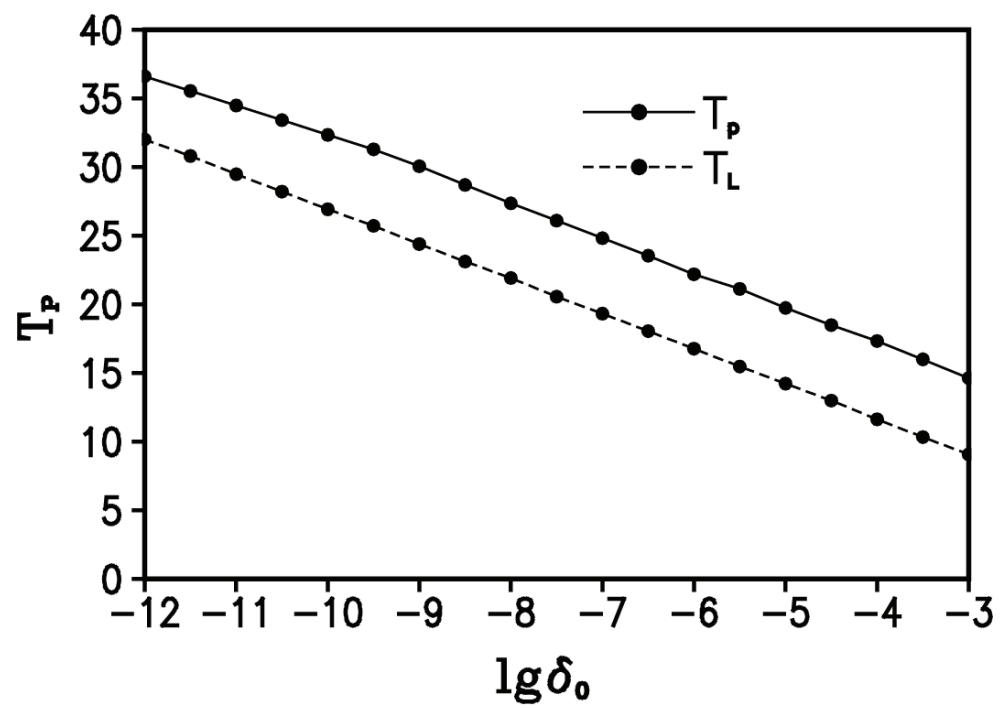

Fig. 6. Same as Fig. 4, but for the Lorenz63 model.

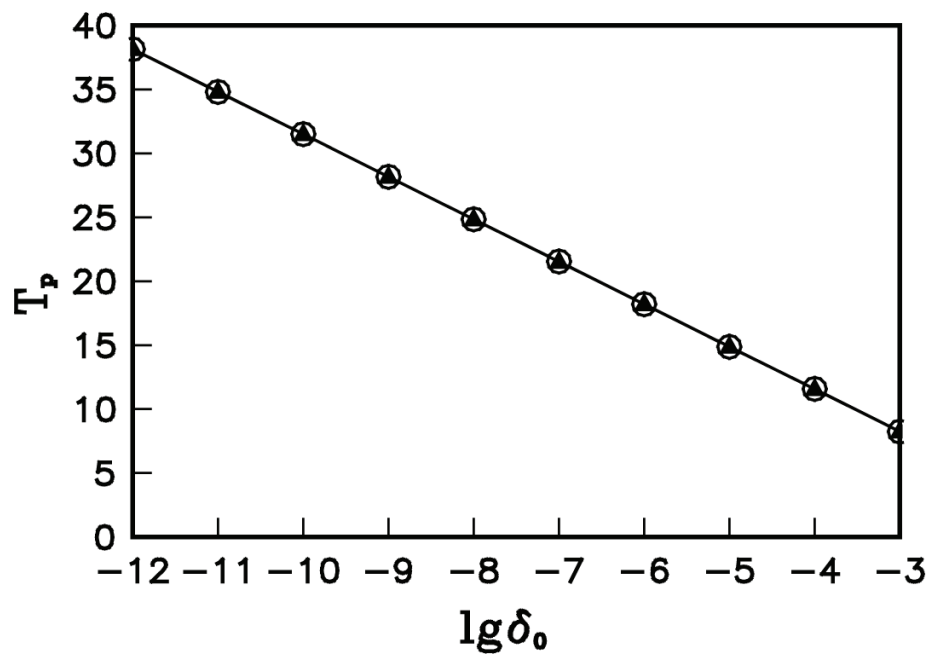

Fig. 7. Predictability limits obtained from Eq. (17) (open circles) and those obtained using the NLLE approach (closed triangles) in the logistic map as a function of $\delta_{0}$ of various magnitudes. 


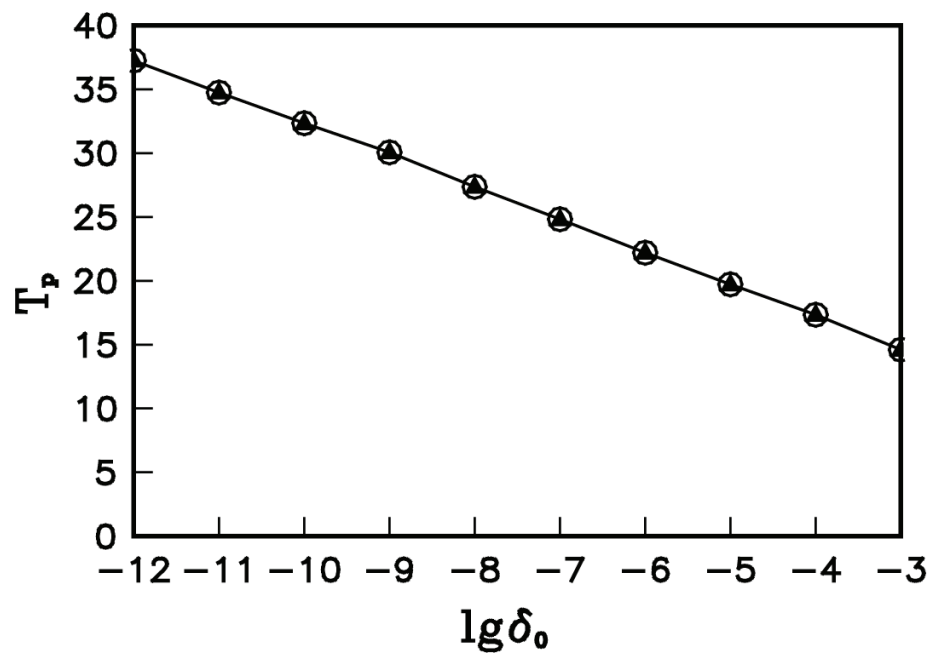

Fig. 8. Same as Fig. 7, but for the Lorenz63 model.

\section{References}

[1] E. N. Lorenz, J. Atmos. Sci. 20 (1963) 130.

[2] E. N. Lorenz, J. Atmos. Sci. 26 (1969) 636.

[3] J. P. Eckmann, D. Ruelle, Rev. Mod. Phys. 57 (1985) 617.

[4] Z. Toth, Mon. Wea. Rev. 119 (1991) 65.

[5] W. Y. Chen, Mon. Wea. Rev. 117 (1989) 1227.

[6] V. Krishnamurthy, J. Atmos. Sci. 50 (1993) 2215.

[7] R. M. May, Nature 261 (1976) 459.

[8] E. N., Lorenz, in Proceedings of a Seminar Held at ECMWF on Predictability (I), European Centre for Medium-Range Weather Forecasts, Reading, UK, 1996, p. 1.

[9] J. M. Nese, Physica D 35 (1989) 237.

[10] E. Kazantsev, Appl. Math. Comp. 104 (1999) 217.

[11] C. Ziemann, L. A. Smith, J. Kurths, Phys. Lett. A 4 (2000) 237.

[12] S. Yoden, M. Nomura, J . Atmos. Sci . 50 (1993) 1531.

[13] J. F. Lacarra, O. Talagrand, Tellus 40A (1988) 81.

[14] M. Mu, W. S. Duan, B. Wang, Nonlinear Process. Geophys. 10 (2003) 493.

[15] J. P. Li, R. Q. Ding, B. H. Chen, in Review and prospect on the predictability study of the atmosphere, Review and Prospects of the Developments of Atmosphere Sciences in Early 21st Century, China Meteorology Press, 96.

[16] R. Q. Ding, J. P. Li, Phys. Lett. A 364 (2007) 396.

[17] R. Q. Ding, J. P. Li, K. J. Ha, Chin. Phys. Lett. 25 (2008) 1919.

[18] C. Rose, M. D. Smith, Mathematical Statistics with Mathematica, Springer-Verlag, New York, 2002. 
[19] J. Guckenheimer, P. J. Holmes, Nonlinear Oscillations, Dynamical Systems, and Bifurcations of Vector Fields, Springer-Verlag, New York, 1983.

[20] A. Wolf, J. B. Swift, H. L. Swinney, J. A. Vastano, Physica D 16 (1985) 285. 


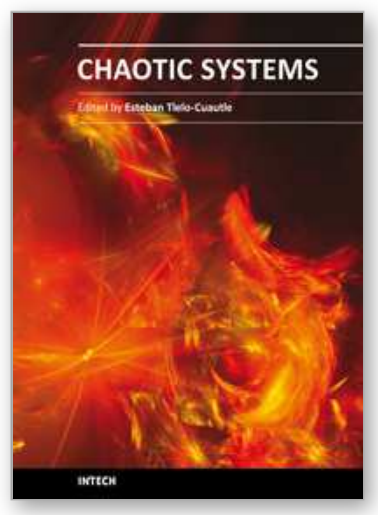

\author{
Chaotic Systems \\ Edited by Prof. Esteban Tlelo-Cuautle
}

ISBN 978-953-307-564-8

Hard cover, 310 pages

Publisher InTech

Published online 14, February, 2011

Published in print edition February, 2011

This book presents a collection of major developments in chaos systems covering aspects on chaotic behavioral modeling and simulation, control and synchronization of chaos systems, and applications like secure communications. It is a good source to acquire recent knowledge and ideas for future research on chaos systems and to develop experiments applied to real life problems. That way, this book is very interesting for students, academia and industry since the collected chapters provide a rich cocktail while balancing theory and applications.

\title{
How to reference
}

In order to correctly reference this scholarly work, feel free to copy and paste the following:

Jianping Li and Ruiqiang Ding (2011). Relationship between the Predictability Limit and Initial Error in Chaotic Systems, Chaotic Systems, Prof. Esteban Tlelo-Cuautle (Ed.), ISBN: 978-953-307-564-8, InTech, Available from: http://www.intechopen.com/books/chaotic-systems/relationship-between-the-predictability-limit-andinitial-error-in-chaotic-systems

\section{INTECH}

open science | open minds

\section{InTech Europe}

University Campus STeP Ri

Slavka Krautzeka 83/A

51000 Rijeka, Croatia

Phone: +385 (51) 770447

Fax: +385 (51) 686166

www.intechopen.com

\section{InTech China}

Unit 405, Office Block, Hotel Equatorial Shanghai

No.65, Yan An Road (West), Shanghai, 200040, China

中国上海市延安西路65号上海国际贵都大饭店办公楼 405 单元

Phone: +86-21-62489820

Fax: +86-21-62489821 
(C) 2011 The Author(s). Licensee IntechOpen. This chapter is distributed under the terms of the Creative Commons Attribution-NonCommercialShareAlike-3.0 License, which permits use, distribution and reproduction for non-commercial purposes, provided the original is properly cited and derivative works building on this content are distributed under the same license. 